\title{
Complexity and Application of Tobacco Manufacturer Pricing Game considering Market Segments
}

\author{
Shubing Guo, ${ }^{1,2}$ Junhai Ma, ${ }^{1}$ and Xueli Zhan ${ }^{3}$ \\ ${ }^{1}$ Group of Nonlinear Dynamics and Chaos, College of Management and Economics, Tianjin University, Tianjin 300072, China \\ ${ }^{2}$ Yantai City Tobacco Monopoly Bureau, Yantai 264000, China \\ ${ }^{3}$ School of Economics, Beijing Wuzi University, Beijing 101149, China
}

Correspondence should be addressed to Xueli Zhan; xuelz20163205@126.com

Received 31 March 2017; Accepted 6 June 2017; Published 31 July 2017

Academic Editor: Hammad Khalil

Copyright (C) 2017 Shubing Guo et al. This is an open access article distributed under the Creative Commons Attribution License, which permits unrestricted use, distribution, and reproduction in any medium, provided the original work is properly cited.

\begin{abstract}
This paper considers price as a strategy using two methods of market segmentation through game theory based on the collected data of several typical cities in Shandong province. Three oligarchs are discussed to analyze the complexity of the market in tobacco system. The effect of different factors on the equilibrium price of Nash equilibrium is discussed. What is more, the variation of parameters drives the system to change to uncertain status, even to bifurcation or chaos. We also discuss the model under normal circumstances, when the three parties jointly take chaos control measures, and its impact on the system. Finally, we clarify chaos control of economic significance. The results indicate that only reasonable pricing strategy is a critical point to achieve maximized profit and maintain orderly operation at the same time, which is a constructive suggestion for tobacco companies' operational strategy.
\end{abstract}

\section{Introduction}

Based on the AHP method and using 20 factors, the results of Zhang et al. [1] showed that $29.82 \%$ of the total area was highly suitable for tobacco production and $17.74 \%$ was unsuitable. To solve the problem where the regular IAHP model cannot describe the probability with an interval number, Xiao et al. [2] carried out the probabilistic distribution of the weights calculation in IAHP. By the study of fuzzy judgment matrices, Zhou and Wei [3] discussed a new method for deriving posterior weight with an application into a real example. In order to determine attribute weights in multiple-attribute decision-making, Cui et al. [4] analyzed the limitations of the present approaches to determining weights of attributes. Based on grey incidence, $\mathrm{Xu}$ and Lv [5] proposed a method for group decision-making. According to fuzzy comprehensive judgment applied to many provinces in practice, Zhong et al. [6] used grey correlation analysis for the comprehensive evaluation. Al-Bayati and Hussein [7] applied the evaluation process to obtain the valuable feedback as its major conclusions for further study. With imprecise data, Kao [8] used DMUs under the framework of DEA. In order to develop the status of tobacco industry, Li [9] discussed a few measures for developing modern tobacco agriculture. From a new perspective, Li et al. [10] gave two methods and gave an analysis to solve the problems that the structure of BP neural network will become complex when samples' data is not preprocessed. By using the grey correlation analysis of the BP neural network, Tang [11] gave a method for improving the network's forecasting accuracy.

With the rise of chaos theory, many scholars are interested in building model with the use of complex system method. Ma and Wang [12] established a closed-loop supply chain and found that the system will become more complicated when the retailers exacerbate competition. Considering that players have different adjustment mechanisms and expectations, $\mathrm{Ma}$ and Guo [13] investigated a dynamic duopoly Cournot game model and studied the influence of adjustment factors on the system. Based on a new supply chain model with supplier and two retailers, J. Ma and X. Ma [14] found that the bullwhip effect is impacted by different factors.

Market segmentation is the procedure which divides into different consumer groups according to certain rule; under this rule the standard variable segments are chosen. After 
selecting the standard variable, consumers are assigned to different groups with certain method. Thus market segmentation approach is the main research method to solve these two issues, which is named segmentation criteria to determine the choice of distribution methods.

For the breakdown of standard variables, it may be single or plural. When there are more variables, the number of market segments will increase exponentially. For example, if there are three values for each variable and the total number of variable is three, then there will be $3^{3}=27$ separations of the market. And when the number of variables is four, there will be $3^{4}=81$ separations of the market. Therefore, the number of variables determines the choice of market segmentation. Considering the demand of the actual needs and the degree of difficulty of the two studies, we have to make suitable decision of variable's quantity.

When it comes to market segmentation, there are many ways, such as conjoint analysis, mixed regression model, factor analysis, fuzzy cluster analysis, AHP, and decision tree algorithm.

Tobacco companies in the competitive market adjust their product price to maximize operation profits. Economic significance of chaos control is to minimize the impact of its price and the price of its own competitors in sales. Thus, the tobacco industry needs to improve sales channels, increasing sales service quality and establishing a good brand image. And then gradually the tobacco industry wins the market through quality and service and also wins more consumers.

Consider the situation where three tobacco companies sell their three tobacco products. These three tobacco companies can be seen as a three-oligarch tobacco market. The three tobacco companies' Nash equilibrium price and the change rate of the price have the influence on the equilibrium state.

In practice, more often with real-time changes in the market, the three parties adjust their prices according to the size of the margin of prices. So we need to model the Nash equilibrium of the three parties' game. Find the Nash equilibrium price for tobacco companies and analyze the complexity of price just like impact on the price of the game and the impact on profits of the game and time series game prices.

Finally, through the research, the reasonable pricing strategy is obtained, which is to maintain the image of the enterprise and the profit balance.

\section{Market Segmentation Based on Conjoint Analysis}

To study simplicity, select a standard variable: product benefits. Market segmentation methods include conjoint and mixed regression model.

Conjoint analysis is a statistical method, which is important for product attribution in the minds of consumers as well as the level of product attributes. For tobacco products, they have different properties, such as aroma, taste, smoke, aftertaste intensity, tar, tobacco, and cigarette paper. Different products have different combinations of attribution and attributing levels; for example, the proportion of white sand tar in Hongtashan, Zhonghua, and other brands of cigarettes
TABLE 1: Hongtashan product attributes.

\begin{tabular}{lc}
\hline & Hongtashan \\
Attribute & Description \\
\hline Raw material & Yuxi \\
Additive & Appropriate \\
Taste & Supple \\
Aroma & Plump \\
Gas & Soft \\
Aftertaste & Clean \\
\hline
\end{tabular}

is higher than the property tar in Zhongnanhai, Pride, and other low-tar brands of cigarettes. Hongtashan product attribute levels and combinations can be expressed as shown in Table 1.

Different cigarette brands have different combinations of attributes and attribute levels. For different consumers, their preferences for product attributes are different too. The same sense of property has different satisfaction level and the core of this difference is the consumer demand preference differences. Therefore, when segmenting the product, we must focus on the importance of product attributes and attribute levels to improve people's unit utility.

In general, when using the joint analysis of the product segment, it is divided into two steps. First, the experience of the importance of product attributes and attribute levels brings the utility of the preliminary estimation and then using cluster analysis methods depending on the importance of product attributes and attribute levels brings the utility, which will be assigned to different consumers market. It is easy to think that the first step in the estimation process and the ability to recognize the limitations of the amount of data may result in unreliable model parameters and the second step is based on the analyzing result of the first step. So the first step in the estimation process will affect the final result. In this paper, a mixed regression model is proposed to improve the estimating process of the first step.

To study the market segmentation mixed regression model, it was firstly introduced by DeSarbo joint analysis and others. In the conjoint analysis, the respondents need to rate the product of a group. DeSarbo scoring data assume that each respondent residual obeys multivariate normal distribution and the variance-covariance matrix estimation. In the evaluation of the relevance of different product respondents, the efficiency of the model decreases with the increase of the estimated parameters, if you assume that the residuals are scoring one dollar data independently distributed, although there will be some decrease in the estimated accuracy. However, you can make the model much more efficient, so the change is worth trying. At this time, although the independent distribution of the residual will sacrifice the accuracy, the model's efficiency will be improved, which is worth trying.

Complete contour method is a more traditional conjoint analysis, in addition to a hybrid approach, self-described method, and that based on the selected method. Compared with the other three methods, complete contour method is 
simple with normal accuracy, which is widely used. Complete contour method comprises the following steps:

(a) Determine the product attributes and each attribute specific level.

(b) Prepare an investigation to determine the product mix.

(c) Make a preference based on the likelihood of respondents to rate the degree of purchase for each product combination.

(d) Use the least-squares method to estimate the effectiveness of the model coefficients.

The basic model is as follows:

$$
U(G)=\sum_{i=1}^{n} \sum_{j=1}^{I_{i}} \alpha_{i j} D_{i j} .
$$

This is a utility model expressing the sum of the utility of representing a portfolio $G$. Product attributes are denoted by $i, i=1,2, \ldots, n$. The horizontal property is denoted by $j$, as $l_{i}$ is the number of horizontal values of the property $i . \alpha_{i j}$ represents utility value attribute, which means the utility of product attributes $i$ get from level $j . D_{i j}$ is a dummy variable; when product attribute $i$ has selected level $j$, its value is 1 ; otherwise, it is 0 .

$I_{i}$ indicates the importance of each attribute $i$. The value for this property at all levels brings a little utility, namely, the difference between the maximum and minimum values, expressed as

$$
I_{i}=\max \left(\alpha_{i j}\right)-\min \left(\alpha_{i j}\right) .
$$

$I_{i}$ represents utility full-pitch; attribute weight $W_{i}$ can represent the whole of its utility from the proportion of the sum of all the properties of the effectiveness of the whole distance; namely,

$$
W_{i}=\frac{I_{i}}{\sum_{i=1}^{n} l_{i}} .
$$

We need to have horizontal joint analysis of $n$ attributes and $l_{i}$ data for each property value. Readily available, the level values to be studied are $\sum_{i=1}^{n} l_{i}$. For $l_{i}$ level values of each property, we need to get a reference, so for the property there is a need to estimate $l_{i}-1$ coefficients. Therefore, there are $C=\sum_{i=1}^{n} l_{i}-n$ estimated model coefficients. Assume that each respondent evaluates the needs of $M$ combinations. Data collected from each respondent are $M$. For each respondent, the linear regression equation can be expressed as

$$
\begin{aligned}
S_{p M}= & \beta_{0 M}+\beta_{1 M} X_{1 p M}+\beta_{2 M} X_{2 p M}+\beta_{3 M} X_{3 p M}+\cdots \\
& +\beta_{C M} X_{C p M}+\delta_{p M} .
\end{aligned}
$$

Model $S_{p M}$ represents respondent $p$ scoring the $M$ case of a portfolio. $X_{1 p M}$ to $X_{C p M}$ represents a combination of different levels. Corresponding $\beta_{1 M}$ to $\beta_{C M}$ are utility coefficients under that level. $\beta_{0 M}$ represents intercept model, as $\delta_{p M}$ are the residuals.

In its estimating coefficients, we use random coefficient model, which overcomes unstable shortcomings in the estimation of OLS regression model caused by fewer coefficients.

In order to express a clear and simple scoring model, it can also be represented as a matrix form: $S_{p}=\theta_{p} X_{p}+\delta_{p}$.

From the previous formula, $\theta_{p}=\left[\begin{array}{llll}\beta_{0 M} & \beta_{1 M} & \beta_{2 M} & \beta_{C M}\end{array}\right]$; $X_{p}=\left[\begin{array}{llll}\beta_{0 p M} & \beta_{1 p M} & \beta_{2 p M} & \beta_{C p M}\end{array}\right]^{-1}$.

The residual vector is $\delta_{p}$; covariance matrix is $\sigma_{p}^{2}$.

Therefore, we can use $\theta_{p}, X_{p}$, and $\sigma_{p}^{2}$ to represent the density function of $p$ respondent's scoring, referred to as $f\left(S_{p} \mid \theta_{k}, X_{p}, \sigma_{p}^{2}\right)$.

As mentioned above, for simplicity, in the estimation of the model, we assume that $\delta_{p}$ followed single normal distribution rather than a multivariate normal distribution. The reasons are already mentioned above. Under this assumption, the density function is expressed as the product of $p$ normal distribution possibilities. Assuming that the whole market is subdivided into $K$ markets, the proportion of each market is $w_{k}$, where $\sum_{k=1}^{K} w_{k}=1$. For one market $k$, we give the model coefficient $\theta_{k}$ and its variances $\sigma_{k}^{2}$; the respondents density function can be expressed as

$$
g\left(S_{p} \mid X_{p}, \varphi\right)=\sum_{k=1}^{K} \pi_{k} f\left(S_{p} \mid \theta_{k}, X_{p}, \sigma_{k}^{2}\right) .
$$

From (5), $\varphi=\left(w_{1} \cdots w_{k}, \theta_{1} \cdots \theta_{k}, \sigma_{1}^{2} \cdots \sigma_{k}^{2}\right)$. In this case, coefficients $\theta_{p}$ and $\theta_{p}^{2}$ at the individual level of the density function model have been replaced by model coefficients $\theta_{k}$ and $\sigma_{k}^{2}$ at the segment level, so that the replacement model is a mixed regression model. With the estimated coefficient $\varphi$, the probability $p$ individual that belongs to the $q$ market can be calculated based on Bayes' formula, which is as follows:

$$
P\left(q \mid X_{p}, S_{p}, \varphi\right)=\frac{\pi_{q} f\left(S_{p} \mid \theta_{q}, X_{p}, \sigma_{q}^{2}\right)}{\sum_{k=1}^{K} \pi_{k} f\left(S_{p} \mid \theta_{k}, X_{p}, \sigma_{k}^{2}\right)} .
$$

Using this method, you can assign each respondent to a segment of the market. The principle is to assign the highest probability of the respondents to its home market. Here we will only divide the respondents to a market rather than assigning them to multiple markets.

Each respondent has $M$ data points; thus the total amount of data is $P \times M$. Based on statistical results, in the case of the model parameter estimation, we use the likelihood function:

$$
\begin{aligned}
L & =\prod_{p=1}^{P} \ln \left[g\left(S_{p} \mid X_{p}, \varphi\right)\right] \\
& =\prod_{p=1}^{P} \sum_{k=1}^{K} \pi_{k} f\left(S_{p} \mid \theta_{k}, X_{p}, \sigma_{k}^{2}\right) .
\end{aligned}
$$


TABLE 2: Consumption data of 26 regions in Shandong province.

\begin{tabular}{|c|c|c|c|c|c|c|c|}
\hline & Clothing & $\begin{array}{c}\text { Household } \\
\text { equipment and } \\
\text { services } \\
\end{array}$ & Healthcare & $\begin{array}{c}\text { Transportation } \\
\text { and } \\
\text { communication }\end{array}$ & $\begin{array}{c}\text { Education, } \\
\text { culture, and } \\
\text { entertainment }\end{array}$ & Living & $\begin{array}{c}\text { Miscellaneous } \\
\text { goods and } \\
\text { services }\end{array}$ \\
\hline Jinan & 924.81 & 536.37 & 793.09 & 1448.33 & 1181.16 & 985.72 & 310.2 \\
\hline Qingdao & 1041.7 & 656.94 & 804.75 & 988.28 & 1435.28 & 951.56 & 286.07 \\
\hline Zibo & 900.05 & 536.36 & 783.44 & 1048.06 & 1108.46 & 1013.74 & 261.81 \\
\hline Zaozhuang & 863.73 & 440.16 & 323.91 & 590.55 & 945.03 & 458.01 & 163.9 \\
\hline Dongying & 1509.12 & 625.28 & 770.32 & 1095.2 & 1842.6 & 751.32 & 410.97 \\
\hline Yantai & 1458.49 & 272.42 & 933.11 & 882.52 & 999.45 & 790.02 & 338 \\
\hline Weifang & 1016.72 & 538.1 & 590.06 & 917.65 & 947.75 & 972.66 & 212.75 \\
\hline Jining & 820.83 & 826.42 & 417.45 & 619.61 & 791.7 & 616.06 & 162.04 \\
\hline Tyan & 798.03 & 443.74 & 538.66 & 623.12 & 1348.09 & 727.06 & 248.7 \\
\hline Weihai & 1369.93 & 609.62 & 682.48 & 1080.86 & 1261.65 & 1018.55 & 542.62 \\
\hline Rizhao & 982.9 & 571.58 & 454.98 & 1195.46 & 825.89 & 579.91 & 277.91 \\
\hline Laiwu & 863.38 & 713.7 & 467.06 & 978.89 & 1009.62 & 714.66 & 183.19 \\
\hline Linyi & 980.49 & 511.48 & 378.77 & 1176.03 & 1070.76 & 854.93 & 240.47 \\
\hline Dezhou & 889.33 & 380.85 & 436.79 & 887.1 & 1160.2 & 594.6 & 224.04 \\
\hline Binzhou & 778.1 & 430.06 & 392.1 & 946.61 & 1272.55 & 672.48 & 191.03 \\
\hline Heze & 804.84 & 336.32 & 367.33 & 516.18 & 633.27 & 436.34 & 183.51 \\
\hline Wendeng & 742.33 & 314.85 & 377.92 & 952.96 & 446.54 & 769.48 & 200.35 \\
\hline Zhucheng & 819.68 & 333.71 & 302.22 & 945.79 & 785.61 & 479.52 & 134.95 \\
\hline Qingzhou & 776.72 & 333.94 & 627.8 & 865.29 & 806.67 & 786.54 & 237.13 \\
\hline Weishan & 929.52 & 694.9 & 351.51 & 598.42 & 1274.28 & 410.42 & 299.99 \\
\hline Linqing & 539.62 & 337.68 & 372.83 & 433.12 & 479.88 & 639.22 & 149.62 \\
\hline Feixian & 629.31 & 402.05 & 199.15 & 450.1 & 757.74 & 371.59 & 61.07 \\
\hline Lijin & 795.41 & 327.8 & 611.97 & 760.38 & 1439.42 & 640.55 & 224.19 \\
\hline Wucheng & 615.4 & 426.88 & 385.36 & 382.57 & 747.51 & 524.08 & 94.81 \\
\hline Donga & 516.72 & 255.33 & 594.04 & 358.94 & 539.33 & 468.61 & 125.64 \\
\hline Juye & 603.64 & 405.99 & 349.08 & 625.14 & 622.33 & 393.37 & 196.59 \\
\hline
\end{tabular}

Multiplicative model is a simple form by taking the logarithm on both sides of the equation.

$$
\begin{aligned}
\ln L & =\sum_{p=1}^{P} \ln \left[g\left(S_{p} \mid X_{p}, \varphi\right)\right] \\
& =\sum_{p=1}^{P} \ln \left[\sum_{k=1}^{K} \pi_{k} f\left(S_{p} \mid \theta_{k}, X_{p}, \sigma_{k}^{2}\right)\right] .
\end{aligned}
$$

The value for market segments is always determined by the Bayesian Information Criterion and the Akaike information criterion. The calculation method for Bayesian Information Criterion is

$$
C_{1}=-2 \ln L+(\ln P) \times R .
$$

Akaike information criterion is calculated as follows:

$$
C_{2}=-2 \ln L+2 R \text {, }
$$

where $R$ represents the number of estimated model coefficients needed, when $C_{1}$ and $C_{2}$ achieve the minimum; corresponding mode value is the number of market segments.

\section{Consumption Region Division with Factor Analysis}

Table 2 shows people's income information and nonfood expenditure statistics in different regions of Shandong province.

In order to study the consumption level of different regions in Shandong Province, we analyze the consumption data of 26 cities or regions in Shandong Province in 2005.

Part of the original data is shown in Table 2.

Firstly make principal component analysis of the data. Original indices and their corresponding attributes are as follows:

$$
\begin{aligned}
& X_{1}: \text { clothing } \\
& X_{2}: \text { household equipment and services } \\
& X_{3} \text { : healthcare } \\
& X_{4} \text { : transport and communications } \\
& X_{5} \text { : education and cultural and recreational services } \\
& X_{6} \text { : living } \\
& X_{7} \text { : miscellaneous goods and services }
\end{aligned}
$$


TABLE 3: Total variance explained.

\begin{tabular}{|c|c|c|c|c|c|c|c|c|c|}
\hline \multirow{2}{*}{ Component } & \multicolumn{3}{|c|}{ Initial eigenvalues } & \multicolumn{3}{|c|}{ Extraction sums of squared loadings } & \multicolumn{3}{|c|}{ Rotation sums of squared loadings } \\
\hline & Total & $\%$ of variance & Cumulative\% & Total & $\%$ of variance & Cumulative\% & Total & $\%$ of variance & Cumulative $\%$ \\
\hline 1 & 4.206 & 60.085 & 60.085 & 4.206 & 60.085 & 60.085 & 2.518 & 35.971 & 35.971 \\
\hline 2 & .984 & 14.055 & 74.140 & .984 & 14.055 & 74.140 & 2.200 & 31.429 & 67.400 \\
\hline 3 & 681 & 9.724 & 83.864 & .681 & 9.724 & 83.864 & 1.152 & 16.463 & 83.864 \\
\hline 4 & .446 & 6.376 & 90.240 & & & & & & \\
\hline 5 & .375 & 5.359 & 95.599 & & & & & & \\
\hline 6 & .176 & 2.517 & 98.115 & & & & & & \\
\hline 7 & .132 & 1.885 & 100.000 & & & & & & \\
\hline
\end{tabular}

Extraction method: principal component analysis.

Using SPSS data analysis software for analysis, prior to principal component analysis, due to the different units of data, direct dimension transformation may have a negative impact on the analysis results. And data obtained is shown in Table 3.

Not difficult to see from the table, the cumulative contribution rate of the first component is $60.085 \%$, while the contribution of the first three components was $83.864 \%$. It can be seen that the first three factors explain more than $70 \%$ of the variance. The three principal components extracted are set to $Y_{1}, Y_{2}$, and $Y_{3}$.

Easy to see for the first principal component, $Y_{1}, X_{1}$, $X_{5}$, and $X_{7}$ have more contribution; for the second principal component, $Y_{2}, X_{4}$, and $X_{6}$ have more contribution; for the third main component, $X_{2}$ has the highest contribution rate.

According to the ingredient matrix after rotation, we can conclude that the expression of the three main components is as follows:

$$
\begin{aligned}
Y_{1}= & 0.827 X_{1}+0.208 X_{2}+0.614 X_{3}+0.274 X_{4} \\
& +0.810 X_{5}+0.273 X_{6}+0.780 X_{7} \\
Y_{2}= & 0.361 X_{1}+0.137 X_{2}+0.622 X_{3}+0.796 X_{4} \\
& +0.131 X_{5}+0.903 X_{6}+0.446 X_{7} \\
Y_{3}= & 0.156 X_{1}+0.929 X_{2}-0.218 X_{3}+0.282 X_{4} \\
& +0.332 X_{5}+0.089 X_{6}+0.140 X_{7} .
\end{aligned}
$$

The main ingredient formula has been set with the variables for each region obtaining the value of the main component into each region, $Y_{1}, Y_{2}$, and $Y_{3}$. The value of the main component into each region is shown in Table 4.

We use the variance contribution rate as weight, and the score model can be expressed as

$$
S=0.60085 Y_{1}+0.14055 Y_{2}+0.09724 Y_{3} .
$$

Data analysis software SPSS statistical functions and so forth can be used to calculate the level of consumption in different regions and contribute to market segmentation.

The sales strategy of the product is closely related to the local consumption level. It is necessary to have a proper change strategy in areas where consumption levels are low. What is more, companies need to seriously consider issues.
TABLE 4: The value of the main component into each region.

\begin{tabular}{lccc}
\hline & $Y 1$ & $Y 2$ & $Y 3$ \\
\hline Jinan & 3227.98 & 3236.698 & 1401.396 \\
Qingdao & 3408.522 & 2928.147 & 1477.314 \\
Zibo & 3002.92 & 2897.338 & 1258.334 \\
Zaozhuang & 2184.902 & 1654.14 & 1017.032 \\
Dongying & 4169.335 & 3084.489 & 1693.371 \\
Yantai & 3366.445 & 2841.781 & 975.5045 \\
Weifang & 2765.644 & 2635.576 & 1219.652 \\
Jining & 2212.662 & 1894.687 & 1319.879 \\
Tyan & 2738.167 & 2123.986 & 1142.111 \\
Weihai & 3698.176 & 3189.965 & 1521.553 \\
Sunshine & 2582.717 & 2423.515 & 1286.979 \\
Laiwu & 2573.238 & 2338.466 & 1396.388 \\
Linyi & 2760.328 & 2615.265 & 1342.437 \\
Texas & 2602.786 & 2139.871 & 1116.959 \\
Binzhou & 2596.418 & 2196.353 & 1211.457 \\
Heze & 1877.739 & 1534.8 & 778.2524 \\
Wendeng & 1900.588 & 2147.431 & 839.4313 \\
Zhucheng & 2064.511 & 1878.562 & 961.1083 \\
Qingzhou & 2387.455 & 2327.088 & 909.5647 \\
Weishan & 2671.25 & 1797.075 & 1384.279 \\
Linqing & 1544.009 & 1524.539 & 675.9059 \\
Feixian & 1612.519 & 1226.46 & 848.3813 \\
Lijin & 2825.749 & 2184.929 & 1075.911 \\
Wucheng & 1761.671 & 1438.315 & 824.54 \\
Donga & 1606.313 & 1426.568 & 527.8837 \\
Juye & 1734.097 & 1512.691 & 840.6687 \\
\hline & & & \\
& & & \\
\hline
\end{tabular}

Not difficult to see from the above analysis, different levels of consumption require different sales focus. For example, the consumption level is arranged in the top three in Dongying, Weihai, and Qingdao. For Dongying City, consumption levels are somewhat used to dress the cultural and recreational projects; for Qingdao, the consumer inclines more to education. Tobacco products should belong to miscellaneous goods and services, so for tobacco companies, when considering the level of consumption of the country and developing marketing strategies, they should not only consider the total level of consumption of the country but also take into 
consideration the level of consumption for miscellaneous goods.

Product marketing not only needs to consider the local spending power, but also needs to consider the following factors.

(a) Socioeconomic Factors. As a result of the rapid economic development, people's living standards continue to improve. The consumption level in the region with low consumption levels has high room for improvement. It is necessary to take into account the future business opportunities in the development of sales strategy. Companies should stand high and see far ahead with sufficient strategic vision for the job.

(b) Product Quality. Should we provide low quality goods in areas with low consumption levels? The answer of course is no. Low-quality products can indeed reduce costs and drive down selling prices. In fact, consumers find that getting poor quality products can cause irreversible damage to the image of the business.

(c) Consumption Habits. The level of consumption is based on the characteristics of the study area to examine consumer groups and product positioning, but the issue of personal spending habits cannot be ignored. It is difficult for us to meet all the individual needs of consumers, but we cannot view consumers as a static group with specific consumer preferences. With the improvement of living standards, consumers must have higher requirements for the products; for example, the health risks of tobacco products may become increasingly valued. Tobacco companies must respect the habits of consumers in marketing.

The level of consumption in the country to some extent can help make the right business regional marketing strategy with not too hasty making the business decision, which should refine consumer groups, pay attention to product quality, and have a broader strategic vision.

\section{Tobacco Manufacturer Pricing Considering Market Segments}

Three tobacco companies sell three tobacco products. These three tobacco companies can be seen as representing tobacco market with three oligarchs. The competition of three tobacco companies makes the market price eventually enter the Nash equilibrium.

4.1. Tobacco Nash Equilibrium Price. Composition market demand function is

$$
\begin{aligned}
& q_{1}=a_{1}-b_{1} p_{1}+c_{1} p_{2}+d_{1} p_{3}, \\
& q_{2}=a_{2}-b_{2} p_{2}+c_{2} p_{3}+d_{2} p_{1}, \\
& q_{3}=a_{3}-b_{3} p_{3}+c_{3} p_{1}+d_{3} p_{2} .
\end{aligned}
$$

All model coefficients are greater than zero. In addition to the fact that the demand for a fixed price is not affected, along with tobacco company's own price increasing, demand decreases with the increase in the price of other brands; otherwise the demand increases. In addition, the impact of own price changes on demand is greater than the impact of competing prices changes. That is, $b_{1}>c_{1}$ and $b_{1}>d_{1}$. Assume that the sum of all costs for per tobacco product is $w$; then three tobacco companies' profit function can be expressed as

$$
\begin{aligned}
& \pi_{1}=\left(a_{1}-b_{1} p_{1}+c_{1} p_{2}+d_{1} p_{3}\right)\left(p_{1}-w\right), \\
& \pi_{2}=\left(a_{2}-b_{2} p_{2}+c_{2} p_{3}+d_{2} p_{1}\right)\left(p_{2}-w\right), \\
& \pi_{3}=\left(a_{3}-b_{3} p_{3}+c_{3} p_{1}+d_{3} p_{2}\right)\left(p_{3}-w\right) .
\end{aligned}
$$

According to Nash equilibrium, after the three parties in the game, the price reached a steady state, so that the three parties are reluctant to change the price again. So the state corresponding to the price for the marginal profit is zero state. This state may be a function of the price of the derivative profits obtained. The equations to calculate the equilibrium price are therefore

$$
\begin{aligned}
& a_{1}-2 b_{1} p_{1}+c_{1} p_{2}+d_{1} p_{3}+b_{1} w=0, \\
& a_{2}+d_{2} p_{1}-2 b_{2} p_{2}+c_{2} p_{3}+b_{2} w=0, \\
& a_{3}+c_{3} p_{1}+d_{3} p_{2}-2 b_{3} p_{3}+b_{3} w=0 .
\end{aligned}
$$

Solutions of equations can obtain tripartite equilibrium price.

In addition, in order to make the best price existing, the need to make profit function of the second derivative is less than zero; namely,

$$
\begin{aligned}
& \frac{\partial^{2} \pi_{1}}{\partial p_{1}^{2}}=-2 b_{1}<0, \\
& \frac{\partial^{2} \pi_{2}}{\partial p_{2}^{2}}=-2 b_{2}<0, \\
& \frac{\partial^{2} \pi_{3}}{\partial p_{3}^{2}}=-2 b_{3}<0 .
\end{aligned}
$$

For example, we give a set of values for the model simulation. According to the analysis above, the definition, and scope of parameters, make $a_{1}=200, a_{2}=280, a_{3}=200$, $b_{1}=70, b_{2}=90, b_{3}=60, c_{1}=20, c_{2}=10, c_{3}=40, d_{1}=20$, $d_{2}=10, d_{3}=40$, and $w=30$. Numerical simulation is applied to three different tobacco companies. It can be seen that the system has the following characteristics: sales are mainly affected by their own prices and are less affected by the price of competitors. We have studied the specific trends in the following text.

With the use of simulation software for data analysis, we can draw three Nash equilibrium prices: $p_{1}=23.67, p_{2}=$ 19.6, and $p_{3}=31.09$; their profits are $\pi_{1}=2804.82, \pi_{2}=$ 9738.56 , and $\pi_{3}=71.286$. So we give Table 5 to compare the three tobacco companies.

It can be seen that the parameters of their own caused a greater impact and the parameters of the competitors caused a smaller impact. Although the price is low, profits are highest, because the price increase brought about by the increase in sales will increase the balance of profits. 
TABLE 5: The comparison of the three tobacco companies.

\begin{tabular}{lccccc}
\hline Number & Brand image & Affected by own price & Affected by substitute & Equilibrium price & Equilibrium profit \\
\hline 1 & Middle & Middle & Middle & Middle & Middle \\
2 & Good & High & Low & Low & High \\
3 & Middle & Low & High & High & Low \\
\hline
\end{tabular}

4.2. Complexity of Price Game of Three Oligarchic Tobacco Companies. In practice, more often with real-time changes in the market, the three parties adjust their prices according to the size of the margins of prices. When the profit margin is greater than zero, the price increase is conducive to gaining more income, and when the profit margin is less than zero, we need to lower prices in order to obtain greater profits. Therefore, the price adjustment model can be written as

$$
p_{i}(t+1)=p_{i}(t)+g_{i} \times p_{i}(t) \times \frac{\partial \pi_{i}(t)}{\partial p_{i}(t)},
$$

where $g_{i}$ represents the speed of price adjustment of player $i$ $(i=1,2,3)$; then the tripartite price adjustment model is

$$
\begin{aligned}
p_{1}( & +1) \\
= & p_{1}(t) \\
& +g_{1} p_{1}(t)\left(a_{1}-2 b_{1} p_{1}+c_{1} p_{2}+d_{1} p_{3}+b_{1} w\right), \\
p_{2}(t) & 1) \\
= & p_{2}(t) \\
& +g_{2} p_{2}(t)\left(a_{2}+d_{2} p_{1}-2 b_{2} p_{2}+c_{2} p_{3}+b_{2} w\right), \\
p_{3}(t+1) & \\
= & p_{3}(t) \\
& +g_{3} p_{3}(t)\left(a_{3}+c_{3} p_{1}+d_{3} p_{2}-2 b_{3} p_{3}+b_{3} w\right) .
\end{aligned}
$$

We can first obtain the above model Jacobin matrix in order to get a stable domain model:

$$
J=\left|\begin{array}{l}
j_{1}, c_{1} p_{1} g_{1}, d_{1} g_{1} p_{1} \\
d_{2} p_{2} g_{2}, j_{5}, c_{2} g_{2} p_{2} \\
c_{3} g_{3} p_{3}, d_{3} g_{3} p_{3}, j_{9}
\end{array}\right|
$$

From (19),

$$
\begin{aligned}
j_{1}= & 1-2 b_{1} g_{1} p_{1} \\
& +g_{1}\left(a_{1}-b_{1} p_{1}+c_{1} p_{2}+d_{1} p_{3}-b_{1}\left(p_{1}-w\right)\right), \\
j_{5}= & -2 b_{2} g_{2} p_{2} \\
& +g_{2}\left(a_{2}-d_{2} p_{1}-b_{2} p_{2}+c_{2} p_{3}-b_{2}\left(p_{2}-w\right)\right), \\
j_{9}= & -2 b_{3} g_{3} p_{3} \\
& +g_{3}\left(a_{3}-c_{3} p_{1}+d_{3} p_{2}-b_{3} p_{3}-b_{3}\left(p_{3}-w\right)\right) .
\end{aligned}
$$

The characteristic equation of (19) is

$$
|\lambda E-J|=A_{0}+A_{1} \lambda+A_{2} \lambda^{2}+A_{3} \lambda^{3}=F(\lambda)=0,
$$

where

$$
\begin{aligned}
A_{0}= & j_{1} j_{6} j_{8}+j_{2} j_{4} j_{9}+j_{3} j_{5} j_{7}-j_{1} j_{5} j_{9}-j_{3} j_{4} j_{8} \\
& -j_{2} j_{6} j_{7}, \\
A_{1}= & j_{1} j_{5}+j_{1} j_{9}+j_{5} j_{9}-j_{3} j_{7}-j_{2} j_{4}-j_{6} j_{8}, \\
A_{2}= & -j_{1}-j_{5}-j_{9}, \\
A_{3}= & 1 .
\end{aligned}
$$

The Routh-Hurwitz stability criterion proposes that a system whose characteristic roots are both in the unit circle is asymptotically stable, and this equals to satisfying the following conditions:

$$
\begin{aligned}
& F(1)=A_{0}+A_{1}+A_{2}+A_{3}>0, \\
& F(-1)=A_{0}-A_{1}+A_{2}-A_{3}<0, \\
& A_{0}^{2}-A_{3}<0, \\
& \left(A_{3}-A_{0}^{2}\right)^{2}-\left(A_{1}-A_{2} A_{0}\right)^{2}>0 .
\end{aligned}
$$

Large-scale price adjustment will cause great harm to the system, so that the price balance is no longer stable, endangering the stability of the entire system.

In the three vendors' characteristics prescribed value, make

$$
\begin{aligned}
& a_{1}=3.6, \\
& a_{2}=4.2, \\
& a_{3}=3.6, \\
& b_{1}=3.2, \\
& b_{2}=4.2, \\
& b_{3}=2.6, \\
& c_{1}=0.4, \\
& c_{2}=0.2, \\
& c_{3}=0.6, \\
& d_{1}=0.5, \\
& d_{2}=0.3, \\
& d_{3}=0.7, \\
& w=0.15 .
\end{aligned}
$$

Examine the impact of price adjustment factor on the system. 


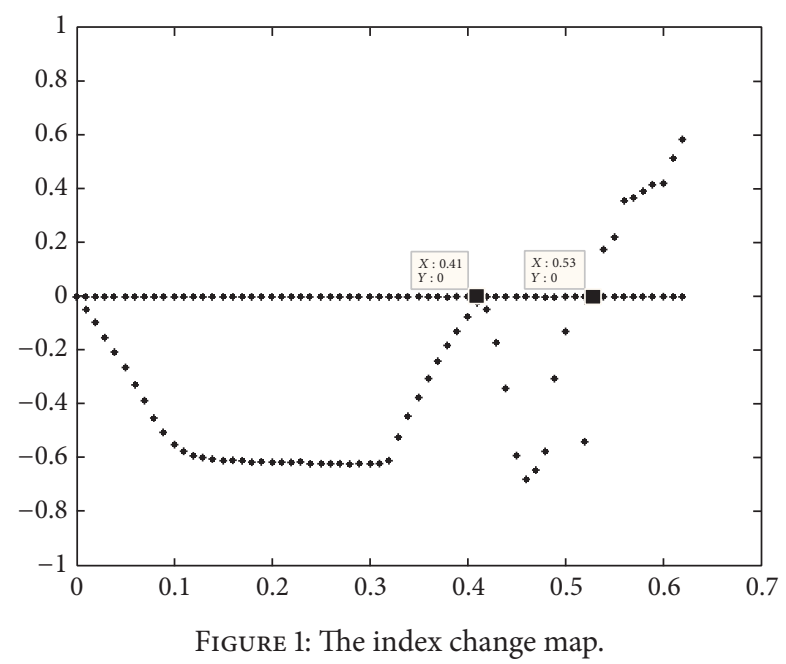

(i) Impact on the Price of the Game. We fixed the adjustment speed of tobacco companies 2 and 3 as 0.1 and studied the impact of the change of the adjustment speed of company 1 on the system.

The size of the index indicates the average convergence or divergence of the system phase space orbit. The index is calculated as

$$
\lambda=\lim _{n \rightarrow \infty} \frac{1}{n} \sum_{n=0}^{n-1} \ln \left|\frac{d f\left(x_{n}, u\right)}{d x}\right| .
$$

The maximum exponent means the mean of the difference after multiple iterations when difference between the initial values is very small. Iterative equation is as follows:

$$
\left|x_{n}-y_{n}\right| \approx\left|\prod_{n=0}^{n-1} \frac{d f\left(x_{n}, u\right)}{d x}\right|_{x_{n}}\left|x_{0}-y_{0}\right| \text {. }
$$

Knowing the index and the number of iterations $n$, we can know the difference between the original minor differences through $n$ iterations. Index may be positive or negative, when the index is positive, it indicates that the difference is a proliferation of state in this direction; when the index is negative, it indicates that the difference within the shrinkage is in that direction. If the system phase space trajectory is away from the equilibrium point, the system is out of stability. Whenever there is a positive index, the system will present chaotic state. Thus, to determine whether there is a system chaotic state, you need to check whether there is a positive index. According to the model and numerical simulation of this article, we can obtain the index change map paper systematically. See Figure 1.

Not difficult to see from the figure, when $g_{1}<0.4$, the maximum is always less than zero, so the system is stable. When $g_{1}=0.41$, the maximum for the first time is zero, because there are no indexes greater than zero, so that the maximum of system does not enter a chaotic state but enters the fold bifurcation state. When $g_{1}>0.53$, the maximum number begins to appear positive; then the system enters a chaotic state.

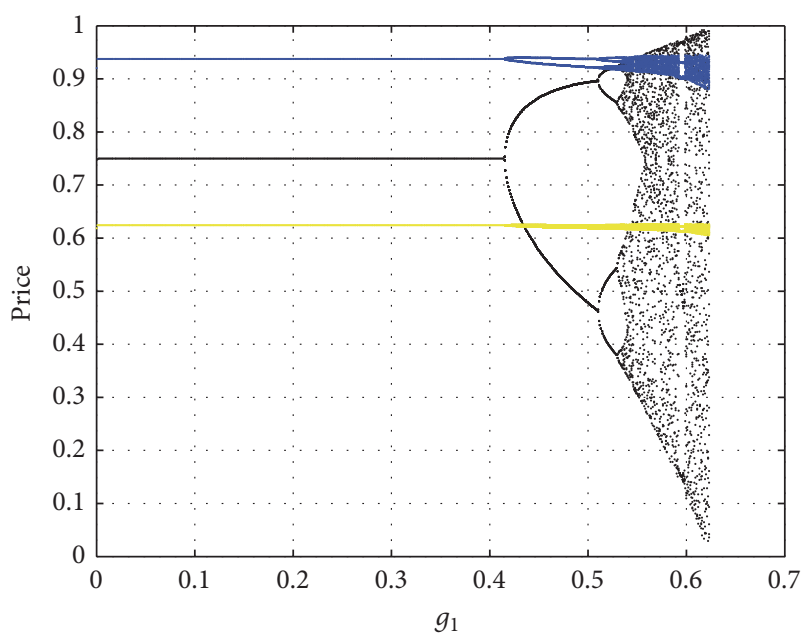

FIgURE 2: The impact of price adjustment speed of evolution of retailer's equilibrium price.

With the increase of retail price adjustment factor 1 , the parties of the dynamic evolution of the equilibrium price are shown in Figure 2.

Under a normal price adjustment system, game player keeps stable in the game after a certain period into a steady state. In this condition, the profit side of the game has reached the maximum, which cannot change unilaterally by the price to obtain greater profits. So that is a Nash equilibrium. But if either one of price adjustment coefficients is too large, it will break the steady state, so that the chaotic system enters a state of disorder. As is shown in Figure 2, when the price adjustment factor is greater than $0.41 \mathrm{~g} 1$, the system began to enter doubling bifurcation state; when $g 1$ is greater than 0.53 , the system enters a chaotic state. In this state, the market competition is chaotic disorder and vicious competition.

(ii) The Impact on Profits of the Game. For the company, the price changes directly affect the acquisition of profits. Therefore, the price of the chaotic state of the game will inevitably lead to chaos game profits. We fixed the adjustment speed of tobacco companies 2 and 3 as 0.1 and analyzed the changes in adjustment speed of tobacco company 1 . Please see Figure 3.

Through two bifurcation diagrams seen in Figure 3, regardless of the nature of the tobacco industry, the impact of the price adjustment factor on the equilibrium price is similar to the acceleration of price adjustment's speed. Tobacco company's prices gradually appear to be chaotic. The rapid rate of price adjustments by either party will lead to significant tripartite profits to chaos. This stability of the market is very negative.

(iii) Time Series Game Prices. Game price time series reflects the increase in the game with the times and the changes in the equilibrium price. At steady state, the price of the game is always in a stable state, as shown in Figure 4.

When the system is in a chaotic state, the price increases with the emergence of the game cycle volatility, as shown in Figure 5. 


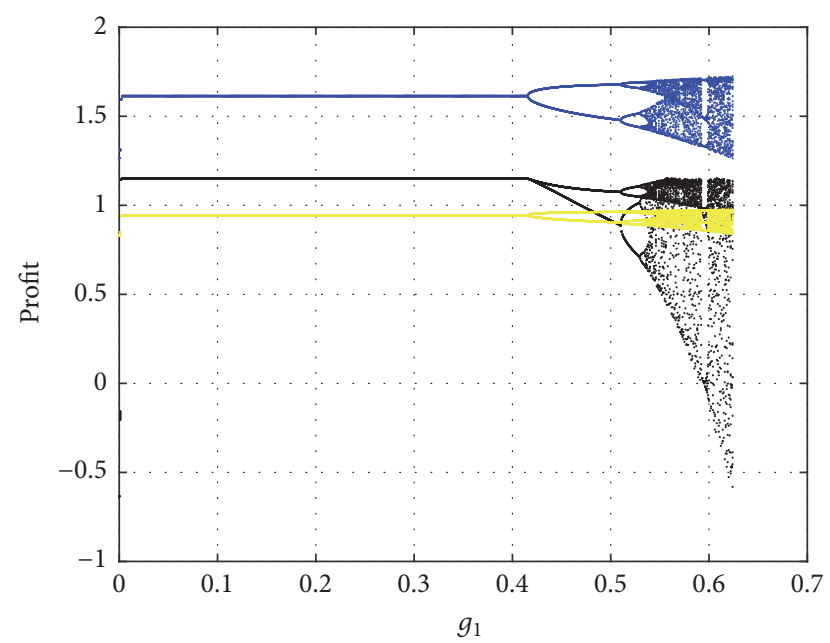

FIGURE 3: The impact of price adjustment speed of evolution of a retailer's balance profit.

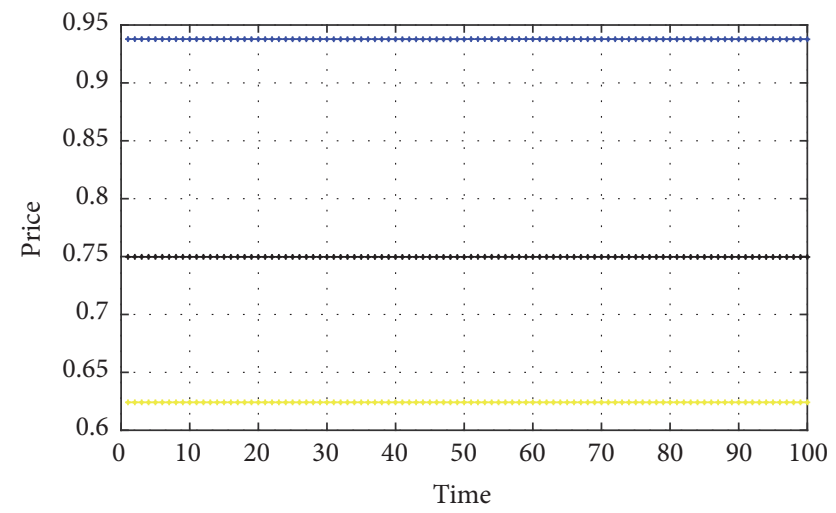

FIGURE 4: The price varies with the steady-state period of the game.

Chaos is a so violent price fluctuations phenomenon, which has certain impact on the stability of the market.

When one of tobacco's price adjustments is too fast, the equilibrium prices go into chaos and the nonlinear feedback control is introduced as follows:

$$
u\left(k, p_{n}\right)=k \prod_{i=1}^{m}\left(p_{n}-p_{i}\right)
$$

After introducing the chaotic control coefficient, the power system of the tobacco company game is shown as follows:

$$
\begin{aligned}
p_{1}(t+1) \\
=p_{1}(t) \\
\quad+g_{1} p_{1}(t)\left(a_{1}-2 b_{1} p_{1}+c_{1} p_{2}+d_{1} p_{3}+b_{1} w\right) \\
\quad+k_{1}\left(p_{1}(t)-p_{1}(t+1)\right),
\end{aligned}
$$

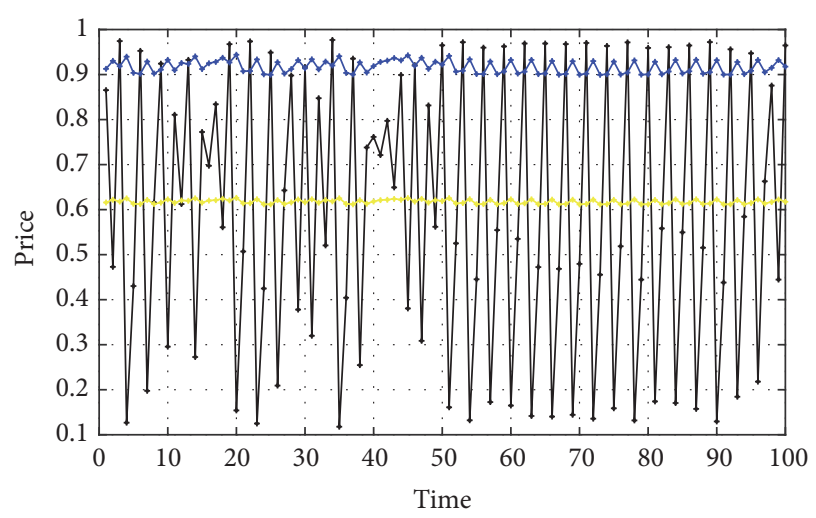

FIGURE 5: The price varies with the chaotic state of the game cycle.

$$
\begin{aligned}
p_{2}(t & +1) \\
= & p_{2}(t) \\
& +g_{2} p_{2}(t)\left(a_{2}+d_{2} p_{1}-2 b_{2} p_{2}+c_{2} p_{3}+b_{2} w\right), \\
p_{3}(t+1) & \\
= & p_{3}(t) \\
& +g_{3} p_{3}(t)\left(a_{3}+c_{3} p_{1}+d_{3} p_{2}-2 b_{3} p_{3}+b_{3} w\right) .
\end{aligned}
$$

According to (28), we can get

$$
\begin{gathered}
p_{1}(t+1)=p_{1}(t)+\left(\frac{g_{1}}{\left(1+k_{1}\right)}\right) p_{1}(t) \\
\cdot\left(a_{1}-2 b_{1} p_{1}+c_{1} p_{2}+d_{1} p_{3}+b_{1} w\right) \\
+k_{1}\left(p_{1}(t)-p_{1}(t+1)\right), \\
p_{2}(t+1)=p_{2}(t)+g_{2} p_{2}(t) \\
\cdot\left(a_{2}+d_{2} p_{1}-2 b_{2} p_{2}+c_{2} p_{3}+b_{2} w\right) \\
+k_{2}\left(p_{2}(t)-p_{2}(t+1)\right), \\
p_{3}(t+1)=p_{3}(t)+g_{3} p_{3}(t) \\
\cdot\left(a_{3}+c_{3} p_{1}+d_{3} p_{2}-2 b_{3} p_{3}+b_{3} w\right) \\
+k_{3}\left(p_{3}(t)-p_{3}(t+1)\right) .
\end{gathered}
$$

The impact of coefficient changes on the stability of the system under such conditions is shown in Figure 6.

It can be seen that the system is stable when the value is close to 0.47 , which means that the tobacco companies can make more rapid price adjustments. 


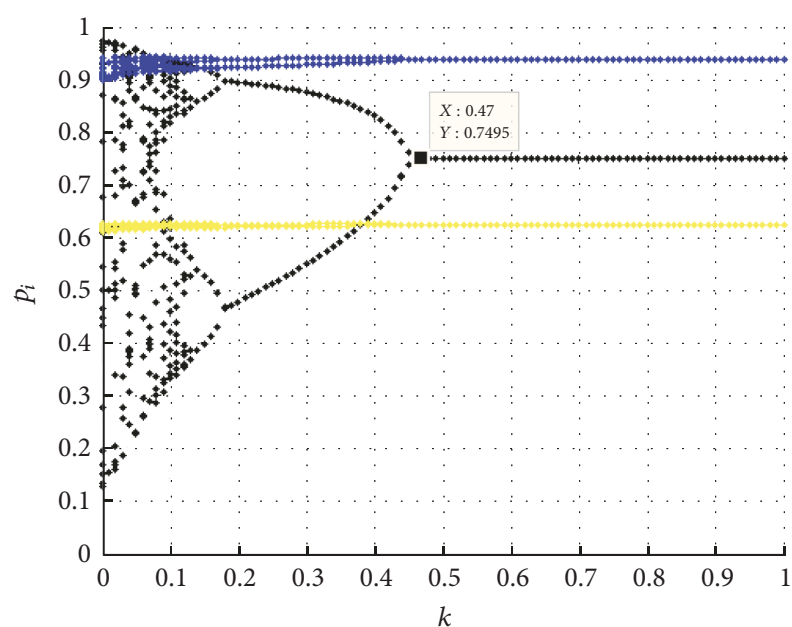

FIGURE 6: Chaos unilateral control factor on system stability.

If all the tobacco companies on the market work together to stabilize the market, chaos control factor of the system becomes

$$
\begin{aligned}
p_{1}(t & +1) \\
= & p_{1}(t) \\
& +g_{1} p_{1}(t)\left(a_{1}-2 b_{1} p_{1}+c_{1} p_{2}+d_{1} p_{3}+b_{1} w\right) \\
& +k_{1}\left(p_{1}(t)-p_{1}(t+1)\right), \\
p_{2}( & +1) \\
= & p_{2}(t) \\
& +g_{2} p_{2}(t)\left(a_{2}+d_{2} p_{1}-2 b_{2} p_{2}+c_{2} p_{3}+b_{2} w\right) \\
& +k_{2}\left(p_{2}(t)-p_{2}(t+1)\right), \\
p_{3}( & +1) \\
= & p_{3}(t) \\
& +g_{3} p_{3}(t)\left(a_{3}+c_{3} p_{1}+d_{3} p_{2}-2 b_{3} p_{3}+b_{3} w\right) \\
& +k_{3}\left(p_{3}(t)-p_{3}(t+1)\right) .
\end{aligned}
$$

According to (30), we can get

$$
\begin{gathered}
p_{1}(t+1)=p_{1}(t)+\left(\frac{g_{1}}{\left(1+k_{1}\right)}\right) p_{1}(t) \\
\cdot\left(a_{1}-2 b_{1} p_{1}+c_{1} p_{2}+d_{1} p_{3}+b_{1} w\right) \\
+k_{1}\left(p_{1}(t)-p_{1}(t+1)\right) \\
p_{2}(t+1)=p_{2}(t)+\left(\frac{g_{2}}{\left(1+k_{2}\right)}\right) p_{2}(t) \\
\cdot\left(a_{2}+d_{2} p_{1}-2 b_{2} p_{2}+c_{2} p_{3}+b_{2} w\right) \\
+k_{2}\left(p_{2}(t)-p_{2}(t+1)\right)
\end{gathered}
$$

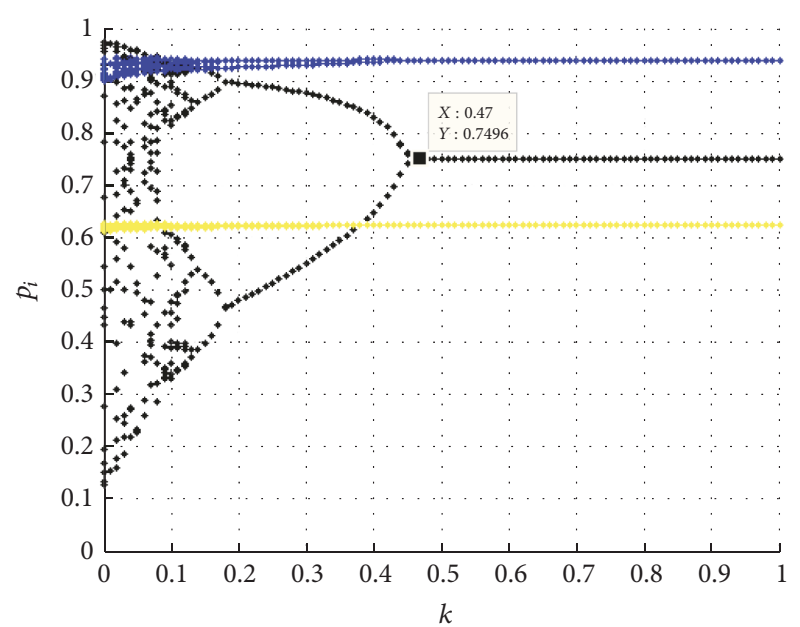

Figure 7: Chaos tripartite control factor on system stability.

$$
\begin{aligned}
& p_{3}(t+1)=p_{3}(t)+\left(\frac{g_{3}}{\left(1+k_{3}\right)}\right) p_{3}(t) \\
& \cdot\left(a_{3}+c_{3} p_{1}+d_{3} p_{2}-2 b_{3} p_{3}+b_{3} w\right) \\
& +k_{3}\left(p_{3}(t)-p_{3}(t+1)\right) .
\end{aligned}
$$

In order to facilitate analysis and comparison, chaos control factor drives to all tobacco companies. Observing chaotic control factor on system stability at this time, see Figure 7.

Under normal circumstances, when the three parties jointly take chaos control measures, the system will be easier to control to enter a stable state. In the diagram, the key point is to make the system's stable $k$ value smaller. In this case, it seems that the three parties take effect to unilateral chaos control when there is not much difference among them. This is mainly due to the price adjustment factor 1 retailers sensitively affected by too high corresponding unilateral system. And the chaotic state of preselected $g 1$ coefficient is relatively large.

Chaos control of economic significance actually reduced by adding $k$ value for each of original price determination coefficients $b, c$, and so on. So its economic significance is actually to minimize the impact on the sales price.

Tobacco companies in the competitive market adjust product price to maximize profits. But the price adjustment speed should not be too fast; otherwise, it will cause the chaotic phenomena in tobacco companies' equilibrium price, which further causes the chaos phenomenon in profits and huge price fluctuations. Therefore, it is necessary to introduce a method of chaos control. Economic significance of chaos control is to minimize the impact of its price and that of competitors in sales. Thus, the tobacco industry needs to improve sales channels and service quality and establish a good brand image. That helps to gradually win the market through quality and service and finally win more consumers. 


\section{Conclusion}

This paper describes two methods of market segmentation. When considering segmentation, the equilibrium price can be determined based on game theory. By using the theory of complexity, we build a three-oligarch competitive model in tobacco market. Lastly, it discusses the stability of the equilibrium price. By numerical simulation, we calculate the stable region with the change of adjustment speed. We also observe the phenomenon of bifurcation and chaos under different values of parameters. In the practice of management, the decision-maker should avoid the price entering into bifurcation and chaos. The reasonable pricing strategy, which is to maintain the image of the enterprise, obtains the profit balance at last. A constructive suggestion is put forward for the development of the tobacco companies.

\section{Conflicts of Interest}

The authors declare that there are no conflicts of interest regarding the publication of this paper.

\section{Acknowledgments}

The research was supported by the Yantai City Tobacco Monopoly Bureau.

\section{References}

[1] J. Zhang, Y. Su, J. Wu, and H. Liang, "GIS based land suitability assessment for tobacco production using AHP and fuzzy set in Shandong province of China," Computers and Electronics in Agriculture, vol. 114, pp. 202-211, 2015.

[2] J. Xiao, F. Z. Luo, C. S. Wang, and Y. Li, "Analysis on probabilistic distribution of weights calculation in the interval-based AHP," Journal of Tianjin University of Science and Technology, vol. 38, no. 3, pp. 189-194, 2005.

[3] Y. F. Zhou and F. J. Wei, "The method for determining the posterior weight of expert based on fuzzy judgment matrices," Chinese Journal of Management Science, vol. 14, no. 03, pp. 71-75, 2006.

[4] J. Cui, Y. G. Dang, and S. F. Liu, "An improved approach for determining weights of attributes in decision making based on grey incidence," Chinese Journal of Management Science, vol. 16, no. 05, pp. 141-145, 2008.

[5] G. L. Xu and Y. J. Lv, "Method for multiple attribute group decision making based on grey incidence," Computer Engineering and Applications, vol. 48, no. 9, pp. 235-237, 2012.

[6] Q. Z. Zhong, R. H. Chen, X. L. Fang et al., "Application of fuzzy comprehensive judgment and gray correlation analysis in tests for newly-introduced peanut varieties," Acta Agriculture Jiangxi, vol. 25, no. 1, pp. 11-14, 2013.

[7] M. A. Al-Bayati and K. Q. Hussein, "Evaluating the efficiency of the instructional websites 'which are oriented to the deaf students' according to the technical criteria," Journal of Convergence Information Technology, vol. 5, no. 2, pp. 138-145, 2010.

[8] C. Kao, "Interval efficiency measures in data envelopment analysis with imprecise data," European Journal of Operational Research, vol. 174, no. 2, pp. 1087-1099, 2006.
[9] J. P. Li, "Consideration on the modern tobacco agriculture development," Journal of Hebei Agricultural Development, vol. 20, no. 9, pp. 108-110, 2008.

[10] J.-M. Li, Y.-H. Hu, and X.-H. Tao, "Recognition method based on principal component analysis and back-propagation neural network," Infrared and Laser Engineering, vol. 34, no. 6, pp. 719723, 2005.

[11] W. M. Tang, "The study of the optimal structure of BP nueral network," Engineering-Theory \& Practice, vol. 10, pp. 95-101, 2005.

[12] J. Ma and H. Wang, "Complexity analysis of dynamic noncooperative game models for closed-loop supply chain with product recovery," Applied Mathematical Modelling. Simulation and Computation for Engineering and Environmental Systems, vol. 38, no. 23, pp. 5562-5572, 2014.

[13] J. Ma and Z. Guo, "The parameter basin and complex of dynamic game with estimation and two-stage consideration," Applied Mathematics and Computation, vol. 248, pp. 131-142, 2014.

[14] J. Ma and X. Ma, "Measure of the bullwhip effect considering the market competition between two retailers," International Journal of Production Research, vol. 55, no. 2, pp. 1-14, 2016. 


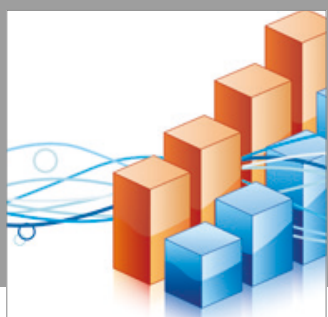

Advances in

Operations Research

vatersals

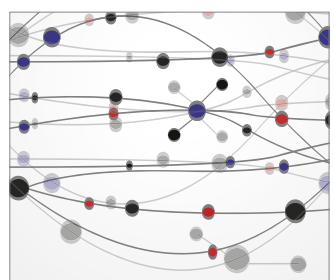

\section{The Scientific} World Journal
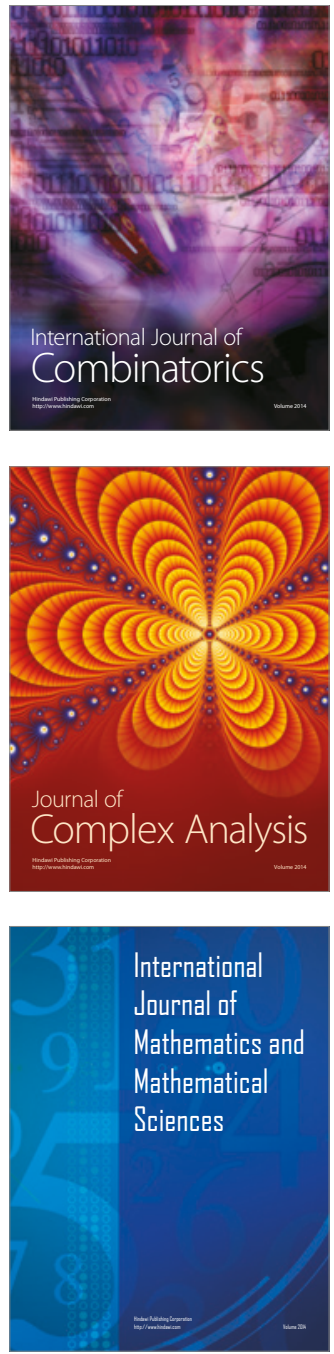
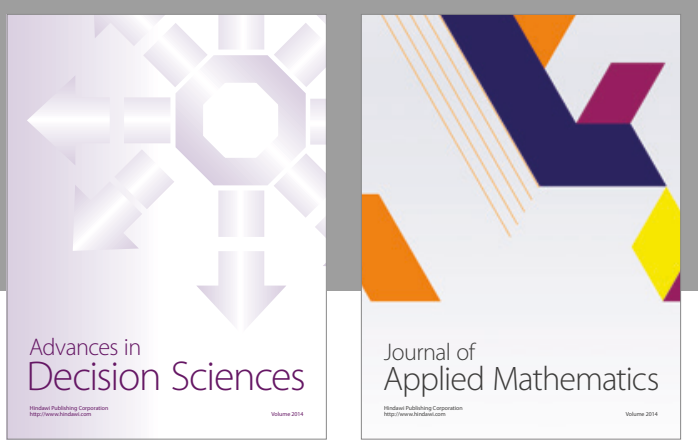

Algebra

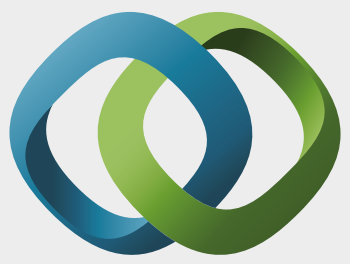

\section{Hindawi}

Submit your manuscripts at

https://www.hindawi.com
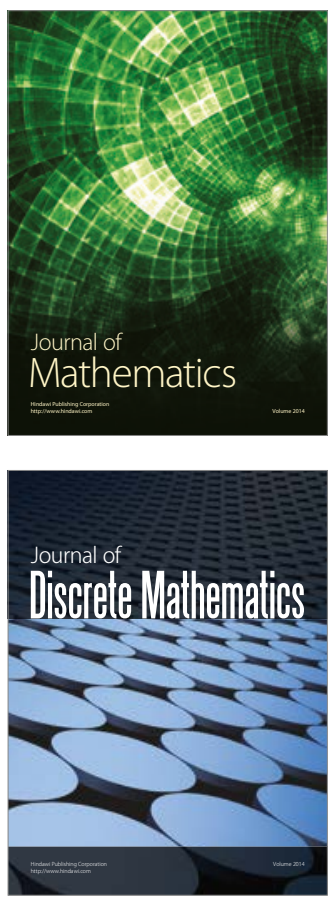

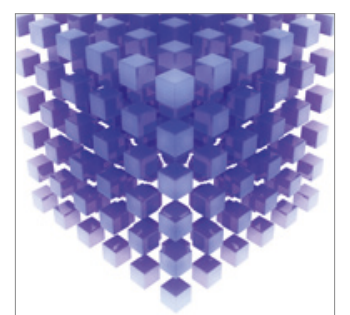

Mathematical Problems in Engineering
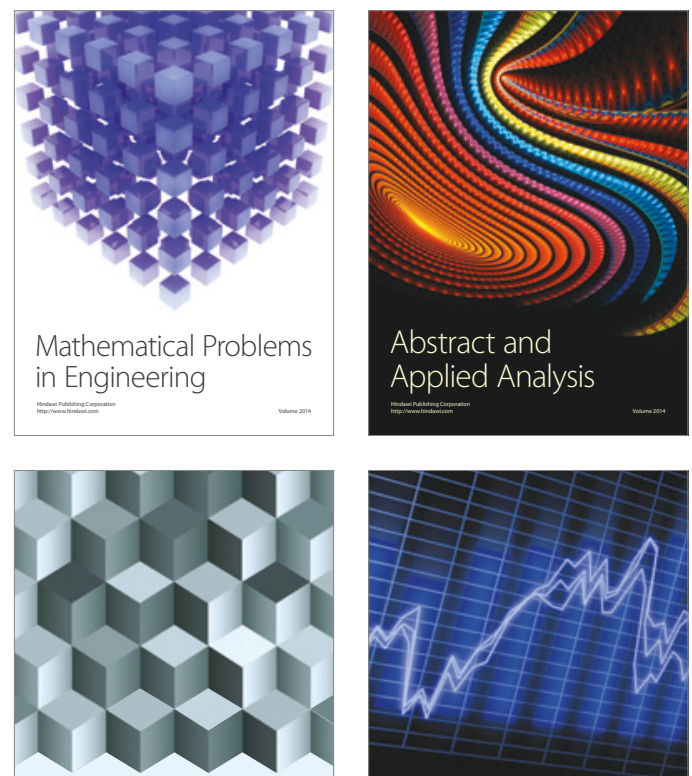

Journal of

Function Spaces

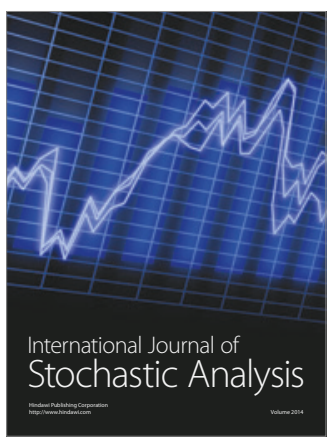

Probability and Statistics
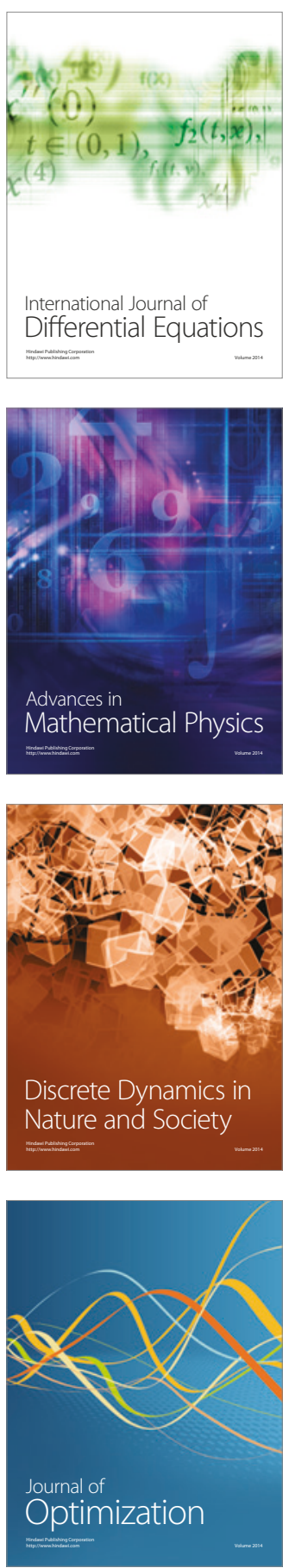